\title{
On the Reliability of the Re/Os Chronometer
}

\author{
J. Conrad* and H. D. Zeh \\ Institut für Theoretische Physik, Universität Heidelberg, Germany \\ Z. Naturforsch. 33a, 887-889 (1978); received May 19, 1978
}

\begin{abstract}
Plausible astrophysical conditions may have substantially increased the rate of ${ }^{187} \mathrm{Re}-\mathrm{decay}$, thereby invalidating the $\mathrm{Re} / \mathrm{Os}$ nuclear chronometer. Moreover, if one assumes that solar system matter has not been exposed to such conditions, then discrepancies between the obtained age of the nuclei and presently accepted Hubble ages appear to exist. These discrepancies prevail with certainty if the $\mathrm{Os} / \mathrm{Re}$ abundance ratio resulting from empirical abundance systematics is accepted instead of the somewhat lower ratio measured for meteorites.
\end{abstract}

The recently measured ratio of the neutroncapture-cross-sections of the isotopes ${ }^{186} \mathrm{Os}$ and ${ }^{187} \mathrm{Os}$ [1] has been used to draw far-reaching conclusions concerning the age of the universe [2]. These conclusions are also based on the ${ }^{187}$ Re halflife and the $\mathrm{Re} / \mathrm{Os}$ abundance ratio [3]. However, as will be discussed below, this abundance ratio and the ${ }^{187}$ Re half-life may not be known as well as is generally assumed. These quantities are uncertain, for reasons in addition to those discussed by other authors $[2,3]$, so that the reliability of the ${ }^{187} \mathrm{Re} /$ ${ }^{187} \mathrm{Os}$ pair as a nucleo-cosmochronometer is still open to discussions [4].

\section{The Elemental Abundance Ratio}

So far Re-age determinations were based on an Os/Re atomic abundance ratio of $12.7 \pm 2$, a value determined for different types of Carbonecous Chondrites (Anders as quoted in Ref. [2]). The most reliable sources for primordial element-abundances are known to be Carbonecous Chondrites of type 1 (C1). The abundance values of $\operatorname{Re}(0.050$ relative to $\left.\mathrm{Si}=10^{6}\right)$ and $\mathrm{Ir}(0.72)$ in C1-Chondrites [5] appear to be reliable, the values for Os vary $[6,7]$ between 0.58 and 0.89 . However, the empirical abundance systematics [8] of primordial $\beta^{-}$(r-process)-nuclei allows to derive rather narrow limits for the Os/Irratio. By using solely the empirical fact that odd-A $\beta^{-}$abundances are never higher than the adjacent even-A $\beta^{-}$abundances (see rule 2 of Ref. [8]) one obtains a lower limit of $\mathbf{1 . 1}$ for this abundance ratio. By also considering the fact that in the regions of the $\beta^{-}$-peaks no odd-even-differences are observed at all, a value of $1.2 \pm 0.1$ is obtained as the most probable one. This leads to a lower limit of 0.8 for the Os-abundance, corresponding to an Os/Re-ratio

* Now at Battelle Institut, Frankfurt, Germany. of 16 . This limit compares well with the one obtained by equating the products $N_{\mathrm{s}} \sigma$ of the s-process abundances and the neutron capture cross sections (rule 1 of Ref. [8]) for $A=176$ (known to be approximately $7.6 \mathrm{mb}$ ) and $A=186$ (using a cross section estimated from Fig. 3 of Ref. [1]). By using only the above limit and the result of the best available laboratory determination for the ${ }^{187} \mathrm{Re}$ half-life [9], viz. $(6.6 \pm 1.3) \times 10^{10} \mathrm{yr}$, one obtains (applying Woosley's [10] correction factor for excited state capture $f=0.82$ ) a lower limit for the mean age of the $\beta^{-}$nuclei of $(23.4 \pm 4.6) \times 10^{9} \mathrm{yr}$, compared to $(19.0 \pm 3.7) \times 10^{9} \mathrm{yr}$ derived from an Os-Re-ratio of 12.7. The first value is hardly compatible with the currently accepted values of the Hubble age [11] which range between 9 and 20 billion years, and which represent upper limits for the age of the universe unless a positive cosmological constant is assumed. The second value for the Re-age could at best be compatible with the highest presently determined value for the Hubble age, and only if an open universe or a positive cosmological constant is assumed. The discrepancy is particularly evident if uniform element synthesis is accepted. The assumption of a sudden synthesis is inconsistent with other age determinations, and a double spike element synthesis with ages of the order of 7 and $20 \times 10^{9} \mathrm{yr}$ might be required.

A reliable determination of the Os abundance in C1-chondrites appears exceedingly valuable. Even if the empirical ratio of $\mathrm{Os} / \mathrm{Re} \approx 12.7$ should be confirmed by additional measurements, interpolation by abundance rules [8] appears to us at least as reasonable as the assumption that $\mathrm{C} 1$-chondrites represent entirely unfractionated primordial matter. Although the conspicuous constancy of the $\mathrm{Os} / \mathrm{Re}$ abundance ratio in different meteorites [12] indeed indicates the lack of chemical fractionation of these elements during meteorite formation, a minor 
degree of fractionation prior to meteorite formation cannot be excluded on empirical grounds. On the other hand, of course, on the basis of the abundance rules, we do not have to exclude the possibility that the $\mathrm{Os} / \mathrm{Re}$ ratio of 12.7 is primordial if the measured C1-abundances of $\mathrm{Re}$ or Ir do not represent primordial values.

A possible way of avoiding the above age discrepancies is discussed in the following section.

\section{The Re-Half-Life}

Clayton [3] emphasized that the life-time of ${ }^{187} \mathrm{Re}$ might be sensitive to thermally induced processes. He suggested as an experimental test to measure the Re isotopic composition in solar wind, but unfortunately this appears not feasible at the present time. If the $\mathrm{K}$-shell is completely ionized the half-life of ${ }^{187} \mathrm{Re}$ is reduced by bound state decay by a factor of approximately $8 \times 10^{3}$, resulting in a half-life of $8 \times 10^{6} \mathrm{yr}[4]$. (Bound state decay into higher shells reduces the half life by less than a factor of 3.) The partial half life for (uniqueforbidden) bound state $\mathrm{K}$-shell decay is given by

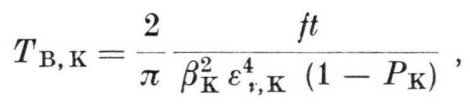

where $\beta_{\mathrm{K}}$ is the $\mathrm{K}$-shell Coulomb amplitude of the bound state electron wave function, $\varepsilon_{\boldsymbol{v}, \mathrm{K}}$ is the neutrino energy and the occupation probability $P_{\mathrm{K}}$ in equilibrium for non-relativistic and non-degenerate electrons is approximately

$$
\begin{aligned}
P_{\mathrm{K}}= & {\left[1+2\left(2 \pi m_{\mathrm{e}} k T\right)^{3 / 2} h^{-3} n_{\mathrm{e}}^{-1}\right.} \\
& \left.\cdot \exp \left\{-I_{\mathrm{K}} / k T\right\}\right]^{-1}
\end{aligned}
$$

with $\mathrm{K}$-shell binding energy $I_{\mathrm{K}}$ and free electron density $n_{\mathrm{e}}$. The accepted end-point-energy [9] $E_{\beta}=2.6 \mathrm{keV}$ leads to a log-ft-value of 11.52 . High degrees of ionization are obtained for high temperatures or low electron densities. However, for electron densities of stellar magnitude ${ }^{187} \mathrm{Os}$ electron capture via excited $187 \mathrm{Os}$ states (the lowest one lying at $9.8 \mathrm{keV}$ ) competes strongly with the temperature-enhanced $\beta$-decay of ${ }^{187} \mathrm{Re}$ (via bound state decay and excited state decay). The total decay rates are given by

$$
\begin{aligned}
\lambda= & Z^{-1} \sum_{i}\left(\lambda_{i}^{\text {bound }}+\lambda_{i}^{\text {cont }}\right)(2 I+1) \\
& \cdot \exp \left\{-E_{i} / k T\right\}
\end{aligned}
$$

where $\lambda_{i}$ are the partial decay rates of the $i$-th state and $Z$ is the nuclear partition function. Using reasonable estimates for the $\log$ - $f t$-values of excited states we have calculated electron densities for which the ratio of the total decay rates $\lambda\left({ }^{187} \mathrm{Re}\right) /$ $\lambda\left({ }^{187} \mathrm{Os}\right)$ is approximately 10 , so that the equilibrium is essentially shifted towards Os, and also those electron densities for which the ${ }^{187}$ Re-decayrate is increased by a factor of 1000. Smaller electron densities further favour Re-decay. These densities are shown in Fig. 1 as functions of tem-

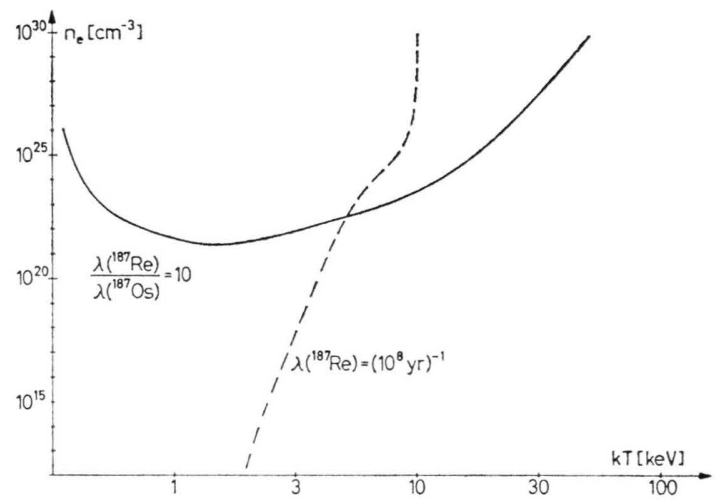

Fig. 1. Upper limits of electron density for which $\lambda\left({ }^{187} \mathrm{Re}\right) /$ $\lambda\left({ }^{187} \mathrm{Os}\right)>10$ (smooth line), and $\lambda\left({ }^{187} \mathrm{Re}\right)>\left(10^{8} \mathrm{yr}\right)^{-1}$ (broken line). Range below both curves strongly increases the rate of effective ${ }^{187} \mathrm{Re}$-decay.

perature. They demonstrate that physical conditions, which invalidate the Re/Os-cosmochronometer are quite plausible, contrary to the assumptions made by Hainebach and Schramm [2], which are based on overconservative estimates by Talbot [13].

Nuclear ages derived by means of the Re/Os. chronometer have therefore to be considered with great caution. Instead of deriving the nuclear age it may be possible to obtain from the ${ }^{187} \mathrm{Re} /{ }^{187} \mathrm{Os}$ abundance ratio a new piece of information on the history of the $\beta^{-}$nuclei after their formation. Since the validity of the shortlived chronometers $129 \mathrm{I}$ and ${ }^{244} \mathrm{Pu}$ as a means to measure the absolute age of the elements has now also been questioned [14], $\mathrm{U}$ and $\mathrm{Th}$ may presently remain the only reliable nuclear chronometers.

We wish to thank Drs. D. D. Clayton and H. E. Suess (who owes this opportunity to a Humboldt award) for valuable help and discussion. 
[1] J. C. Browne and B. L. Berman, Nature London 262 , 197 (1976).

[2] K. L. Hainebach and D. N. Schramm, Atrophys. J. 207, L 79 (1976); Astrophys. J. 212, 347 (1977).

[3] D. D. Clayton, Astrophys. J. 139, 637 (1964); Nature London 224, 56 (1969).

[4] J. Conrad, Thesis, Heidelberg 1976.

[5] U. Krähenbühl, J. W. Morgan, R. Ganapathy, and E. Anders, Geochim. Cosmochim. Acta 37, 1353 (1973).

[6] J. Morgan, in B. Mason, Handbook of Elemental Abundances in Meteorites, Gordon and Breach, New York, 1971, p. 451.

[7] H. Wänke, H. Baddenhausen, H. Palme, and B. Spettel, Earth Planet. Sci. Lett. 23, 1 (1974).
[8] H. E. Suess and H. D. Zeh, Astrophys. Space Sci. 23, 173 (1973).

[9] R. L. Brodzinski and D. C. Conway, Phys. Rev. 138, B 1368 (1965).

[10] S. E. Woosley, J. Holmes, W. A. Fowler, and B. A. Zimmermann, (1976), in preparation.

[11] A. Sandage and G. A. Tamman, Astrophys. J. 210, 7 (1976); G. De Vaucouleurs, in D. S. Evans, External Galaxies and Quasi Stellar Objects, Reidel 1972, p. 353; D. Lynden-Bell, Nature London 270, 396 (1977).

[12] J. W. Morgan and J. F. Lovering, Geochim. Cosmochim. Acta 31, 1893 (1967).

[13] R. J. Talbot Jr., Astrophys. Space Sci. 20, 241 (1973).

[14] D. D. Clayton, Astrophys. J. 199, 765 (1975); M. G. Edmunds, Nature London 267, 393 (1977). 